\title{
Comparison of Sprint Reaction and Visual Reaction Times of Athletes in Different Branches
}

\author{
Murat Akyüz' ${ }^{1}$, Başar Basri Uzaldi², Öznur Akyüz ${ }^{1}$, Yeliz Doğru \\ ${ }^{1}$ School of Physical Education and Sport, Celal Bayar University, Manisa, Turkey \\ ${ }^{2}$ School of Physical Education and Sport, İbrahim Çeçen University, Ağrı, Turkey \\ ${ }^{3}$ School of Physical Education and Sport, Ege University, İzmir, Turkey \\ Correspondence: Öznur Akyüz, School of Physical Education and Sport, Celal Bayar University, Manisa, Halil Erdoğan \\ Cad. Ahmet Bedevi Mah, 45040, Turkey.
}

Received: November 17, 2016

doi:10.11114/jets.v5i1.1987
Accepted: December 1, $2016 \quad$ Online Published: December 14, 2016

URL: http://dx.doi.org/10.11114/jets.v5i1.1987

\begin{abstract}
The aims of this study are to analyse sprint reaction and visual reaction times of female athletes of different branches competing in Professional leagues and to show the differences between them. 42 voluntary female athletes from various branches of Professional leagues of İstanbul (volleyball, basketball, handball) were included in the experiment group of this study. Lafeyetta Moart Reaction meter for visual reaction measurements and Fusionsport Pro Photocell meter for sprint reaction test measurements were used for participating athletes. Mean measurements were obtained for all tests. SPSS 22 statistical package program was used for analysing data for Windows. Kruskall Wallis, Anova and Post Hoc test was used while comparing the data between the groups. Statistical significance level was taken as $\mathrm{p}<0.05$. Study results show that no statistically significant difference was observed among the mean visual reaction values of basketball, volleyball and handball players $(\mathrm{p}>0.05)$. A statistically significant difference was observed among the averages of sprint reaction total values of players $(\mathrm{p}<0.05)$. According to visual reaction measurements, handball players were better compared with the other branches $(\mathrm{p}<0.05)$. No relationship was observed between visual reaction and sprint reactions of players among the branches.
\end{abstract}

Keywords: reaction time, volleyball, basketball, handball

\section{Introduction}

With the level the sport has reached today, increase of competitive conditions, and further specification of qualifications desired in athletes in parallel to that; profile of the athlete at elite level is changing, significant developments are occurring in performance, and development is continuing. The necessity to be the best to compete with opponents in competitions has forced athletes and coaches into new searches and the requirement to benefit from scientific data has become inevitable as a result of such searches (Üstdal and Köker, 1998). Studies conducted for years on detection of sportive skill have shown that sportive skill is a whole of components. Reaction time and hand-eye coordination are a part of such components (Bayar and Koruç, 1992).

Handball, basketball and volleyball require all of the basic motoric properties such as strength, speed, durability, mobility, dexterity and coordination considering duration of game and the necessity to play quickly and correctly. However, as in all team sports; it is seen that coordinative skills such as quick strength and continuity in strength step forward in the struggle given for ball possession. Speed is a physical value, which starts with a stimulant against resistances and forms with the scarcity of the time lapsing for completion of determined movement and travelling of determined distance (Dündar, 2007). Agility is also defined as the body movement performed with speed or change of direction as response to a stimulant. In order for a task to be considered as agility; it must not only contain speed or change of direction but also be an alternating skill, require respond to a stimulant, and must not have been rehearsed in advance (Sheppard and Young, 2006). Tasks, which are considered as agility and include cognitive components (e.g. sprint start of athletes, putting shot), are separated from tasks containing time and space uncertainty (e.g. counter-reaction to spike in volleyball, dodging the opponent in football). Although visual-cognitive components are required to be included in an accurate agility test; aim of agility tests in general is merely to measure the change of direction speed (Sevim, 2006). 
Implementations in sprint practises generally aim at improving acceleration in flat sprint without change of direction, maximum speed and continuity in speed. As flat sprint practises do not have significant effect on running performance at high speed with change of direction, it has also been detected that specific agility practises do not improve flat sprint performance (Young et al., 2001). Hence, relation between flat sprint and agility (running speed with change of direction) is not as strong as expected.

Another important issue related to field and court sports including mixed skills is that performing sprint with a skill (e.g. running with ball and dribbling) increases the complexity of task. As can be understood from the weak relation between flat running skill and the skill to perform tasks such as running with dribbling, increase in complexity affects the athlete's performance (Tsitskarsis et al., 2003). Movement (or reflex) time is the duration from start of movement to end of movement. Accordingly, movement time is the time, which lapses from the moment the athlete starts moving in the start block until he reaches the finish line. Reaction time is the determining factor of performance in many sports. Measuring reaction time is quite complex despite its simple definition. Related sense organs, severity of stimulation, status of the medium, and required stimulation and motivation are several of the factors affecting reaction time (Guyton et al, 2006). While reaction speed workout time lasts for 1 to 3 seconds, movement speed workout time lasts for 3 to 5 seconds. Workouts at maximum tempo lasting for 5 to $10 \mathrm{~m}$ or 1 to 3 seconds are reaction speed improvement workouts and are performed through consumption of the existing ATP in the muscle. In such workouts, excess completion takes places after 0.30 - 1 minute. Hence, $0.30-1 \mathrm{~min}$. active rest must be given after each move in such workouts (Renklikurt, 1974).

This study was performed to examine and reveal the differences of sprint reaction and visual reaction times of female athletes at different branches competing in professional leagues.

\subsection{Literature Review}

A lot of sources published concerning agility include researches in which running and turn directions are known by the athlete. However, productivity of agility movements increases depending on the perception and decision-making process in practise environment. Researches show that elite athletes with high skill level put forward faster and more accurate reaction movement compared to other athletes thanks to the clues they obtain about how movements will occur during practise or game (Aşç1, 2013). General structure of tests, in which agility is evaluated, depends on the measurement of running speed with change of direction on horizontal plane. Scores (change of direction speed and agility), which are measured in tests for evaluation of agility, must be independent of the maximum speed in flat sprint (Sheppard and Young, 2006). Sheppard and Young (2006) have stated that agility is composed of two sub-components as change of direction speed and cognitive factors. According to this definition, agility performance is affected by properties such as flat sprint speed, running technique, anthropometric properties, and leg muscle properties. In addition, it has started to be stated that agility is also associated with components related to change of direction, perceptive and decision-making components due to the fact that changes in direction and speed occur according to the opponent's movements in general. Measurement of agility performance is determined through change of speed tests. For that purpose, zig-zag running speed tests are frequently used in the literature. However, all these tests are closed skill tests since all movements can be planned in advance and no reaction to a stimulant is present. Based on the deficiency of cognitive needs in measurements tests of closed skills, closed skill changes of direction apply different pressures on body compared to open skills (Sheppard and Young, 2006).

Significant contributions of isokinetic dynamometers to evaluation of these muscles have been found with the purpose of maximizing the physical performance of athletes or preventing factors such as injury, which limit their performances, and determining the points to be concentrated during their practises. In their studies, Aagard et al. (2002) showed that use of dynamic control rate reflects the ability to resist the anterior tibial shear forces which occur during maximum quadriceps muscular contractions of hamstrings. Force and strength measurements are said to have effect on change of direction speed; however, this relation can be observed through comparison of directional changes made at short distance (Negrete and Brophy, 2000).

Sprint tests and change of direction speed tests, which include changing direction around stationary objects, are used frequently for measuring agility in sport. According to the results of the studies performed, it can be said that flat spring trainings have limited contribution to the speed of sprint performed with change of direction and flat spring trainings do not increase agility performance. In general, the more changes of direction the less transfer to change of direction speed occurs from flat sprint trainings. It is believed that a strong relation exists between flat sprint speed and the speed of sprint made with change of direction. Despite the fact that these two properties are considered as pair in some studies in the literature, there are no research results to support this view (Sheppard and Young, 2006).

In light of the above information, it is aimed to examine and reveal the differences of sprint reaction and visual reaction times of female athletes at different branches competing in professional leagues. 


\section{Method}

\subsection{Research Model}

A total of 42 athletes participated in this research voluntarily composed of 14 female volleyball players from UPS Sports Club competing in Turkish Women's Volleyball League of 2015-2016 season, 14 female basketball players from Yenibosna Sports Club competing in Turkish Women's Basketball League, and 14 female handball players from Anadolu Üsküdar Sports Club competing in Turkish Women's Handball League Participants were chosen through one-on-one interviews or leaflets and their written consents were received. The study was conducted in accordance with the principles of Helsinki Declaration.

Tests and measurements were performed in the indoor sports hall of UPS Sports Club, sports hall of Yenibosna Sports Club, and Çamlıca Sports Hall of Anadolu Üsküdar Sports Club. Heights and body weights of all participant athletes of the research were measured and recorded in order. Participants were subjected to visual reaction time test and spring reaction time test respectively at two different days and in the same period of day. Environment where measurements were to be performed was arranged as quiet, airy, and such that athletes would not be distracted. Before the measurements, we ensured voluntary participation by informing the subjects of the aim of the study and tried to increase their level of motivation. During tests, athletes implemented test directives in the best way and were warned to be extremely cautious.

Stature of the athletes were measured without shoes with the wall-mounted measuring tape (Seca 769 Hamburg, Germany). Body compositions of the participants were analysed using bioelectrical impedance analysis method (Tanita 300 MA, Tanita C.O, Tokyo-Japan).

Visual reaction time of participants was measured after the same work environment was provided for all volunteers (Lafeyette Moart, USA). Participants were included in the study one by one and informed of the importance of the test to be performed. 5-repetition exercise test was implemented before the test. Eyes were focused on the screen and head was ensured to be held $40 \mathrm{~cm}$, and hands $20 \mathrm{~cm}$ behind the screen. Volunteers were asked to keep their dominant index finger slightly on the button. After the ready command was given, they were asked to press the warning button with an interval of 2.5 seconds and to press the button as soon as they saw the light. 10-repetition measurements of all participants were taken and their arithmetic mean was calculated by taking the best and the worst values.

Reaction time and agility properties of the participants of the study were tested (SmartSpeed, FusionSport, Australia). All data were automatically recorded in the hand computer through timing gates operating via wireless system and remote hand computer, and later installed in computer and rendered ready for statistical analyses.

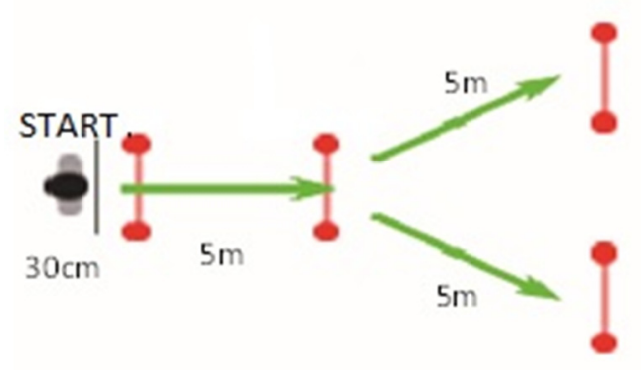

Figure 1. Sprint reaction speed test parkour with change of direction

As provided in Figure 1, participants started the test $30 \mathrm{~cm}$ behind the first gate and ran $5 \mathrm{~m}$ flat sprint with the light stimulant from gates. Then, they completed the test by changing direction according to the light stimulants coming from the right or left gate, which were positioned to create 90 degrees of angles $5 \mathrm{~m}$ ahead of the second gate. The delay time between passing of the middle gate and arrival of the light stimulant was determined automatically by the system as 40 $-45 \mathrm{~ms}$. The probability of all sprints being on the same side throughout that test duration was determined to be less than $1 \%$ due to the fact that change of direction was not under the control of the researcher. Throughout the protocol period, participants were provided with the necessary warnings not to make assumptions regarding from which final gate light would come and not to reduce their speed before passing the final gate, and to run as fast as they could by giving reaction to the light stimulant. 2-repetition measurements of all participants were taken and their arithmetic mean was calculated by taking the best and the worst values.

Data obtained at the end of the study were evaluated using the SPSS package program (version 22.0). In the parameters measured in the research, Kolmogorov-Smirnov and Shapiro-Wilk tests were used for normality assumption, Kruskal 
Wallis and Anova test for variation, and Post Hoc test for multiple comparison. Relations between the variables were evaluated through Pearson correlation analysis. Statistical significance was defined at $\mathrm{p}<0.05$ level.

\subsection{Findings}

General properties of the participants are provided in Table 1. Visual reaction values of the athletes did not display normal distribution as a result of the Kolmogorov-Smirnov and Shapiro-Wilk tests for normality assumption in the parameters. Sprint reaction 1st measurement, sprint reaction 2 nd measurement and sprint reaction total values display normal distribution in the normality test regarding the athletes' sprint reaction values.

Table 1. General characteristics of participants at baseline

\begin{tabular}{lllllll}
\hline & \multicolumn{2}{l}{ Volleyball $(\mathrm{n}=14)$} & \multicolumn{2}{l}{ Basketball $(\mathrm{n}=14)$} & \multicolumn{2}{l}{ Handball $(\mathrm{n}=14)$} \\
\cline { 2 - 7 } Parameters & Median & Min Max & Median & Min Max & Median & Min Max \\
\hline Age $(\mathrm{yr})$ & 17.5 & $15-22$ & 36.2 & $31-40$ & 22.14 & $16-32$ \\
Height $(\mathrm{cm})$ & 174 & $160-188$ & 177 & $163-188$ & 171 & $156-185$ \\
Body weight $(\mathrm{kg})$ & 62.79 & $54-73$ & 82.55 & $61-98.7$ & 61.43 & $49-70$ \\
\hline
\end{tabular}

Table 2. Visual Reaction and Sprint Reaction Relations of Athletes

\begin{tabular}{lllll}
\hline Parameters & & $\begin{array}{l}\text { Basketball }(\mathrm{n}=14) \\
\text { VVisual Reaction } \\
\text { mean } \pm \text { SD }\end{array}$ & $\begin{array}{l}\text { Volleyball }(\mathrm{n}=14) \\
\text { Mean } \pm \text { Seaction }\end{array}$ & $\begin{array}{l}\text { Handball }(\mathrm{n}=14) \\
\text { Visual Reaction } \\
\text { mean } \pm \text { SD }\end{array}$ \\
\cline { 3 - 5 } Reaction 1st & $\mathrm{r}$ & -.241 & -.282 & -.382 \\
Measurement & $\mathrm{p}$ & .407 & .329 & .178 \\
Sprint & $\mathrm{r}$ & -.052 & -.138 & .089 \\
Reaction & $\mathrm{p}$ & .859 & .638 & .761 \\
2nd Measurement & $\mathrm{r}$ & -.136 & -.089 & -.051 \\
Sprint & $\mathrm{p}$ & .643 & .762 & .863 \\
Reaction & & & & \\
Total & & & & \\
\hline
\end{tabular}

$\mathrm{p}<0.05$

When the relation between the visual reaction measurements and sprint reaction values of basketball players are examined, there is no relation between the visual reactions and sprint reactions of basketball players. When the relation between the visual reaction measurements and sprint reaction values of handball players are examined, there is no relation between the visual reactions and sprint reactions of handball players. When the relation between the visual reaction measurements and sprint reaction values of volleyball players are examined, there is no relation between the visual reactions and sprint reactions of volleyball players (Table 2).

Table 3. Mean Differences of Athletes' 1st Measurement Values and Total Measurement Values

\begin{tabular}{llllll}
\hline & \multicolumn{2}{l}{ Sprint Reaction 1st Measurement } & \multicolumn{3}{l}{ Sprint Reaction Total } \\
\hline $\begin{array}{l}\text { (I) Branches of } \\
\text { Athletes }\end{array}$ & $\begin{array}{l}\text { (J) Branches of } \\
\text { Basketball }\end{array}$ & $\begin{array}{l}\text { Mean Differences } \\
\text { (I-J) }\end{array}$ & $\mathrm{p}$ & Mean Differences (I-J) & $\mathrm{p}$ \\
& Handball & -55.14 & .462 & -144.71 & .347 \\
Handball & Volleyball &.$-134.79^{*}$ & .015 & $.280 .07^{*}$ & .025 \\
& Basketball & 55.14 & .462 & 144.71 & .347 \\
Volleyball & Volleyball & -79.64 & .207 & -135.36 & .394 \\
& Basketball & $.134 .79 *$ & .015 & $.280 .07 *$ & .025 \\
& Handball & 79.64 & .207 & 135.36 & .394 \\
\hline
\end{tabular}

When the Post Hoc Test regarding the mean differences of athletes' sprint reaction 1st measurement values is examined, sprint reaction 1st measurement means of basketball players and volleyball players are different from each other. Sprint reaction mean values of volleyball players are greater than basketball players' mean sprint reaction 1 measurement values. Mean sprint reaction total measurement values of basketball players and volleyball players are different from each other. Sprint reaction total measurement mean values of volleyball players are greater than basketball players' sprint reaction total measurement mean values (Table 3).

When the Anova test regarding the mean differences of the athletes' sprint reaction 1st measurement values is examined, there are differences between the athletes' sprint reaction 1st measurement mean values and their branches $(\mathrm{p}<0.05)$. When the Anova test regarding the mean differences of the athletes' sprint reaction 2 nd measurement values is examined, there are differences between the athlete's sprint reaction 2 nd measurement mean values and their branches $(p<0.05)$. Athletes' spring reaction total mean values are different from each other $(p<0.05)$. In other words, it was seen that the basketball players' sprint reaction mean values were the best, while the volleyball players' were the worst (Table 4). 
Table 4. Mean Differences of Athletes' 1st Measurement Values, 2nd Measurement Values and Sprint Reaction Means

\begin{tabular}{|c|c|c|c|c|c|c|c|c|c|c|}
\hline & & $\begin{array}{l}\text { Sprint React } \\
\text { 1st Measure }\end{array}$ & & & $\begin{array}{l}\text { Sprint } \\
\text { Measurement }\end{array}$ & Reaction & 2nd & Sprint React & n Total & \\
\hline Variables & $\mathrm{N}$ & Median & $\mathrm{F}$ & $\mathrm{p}$ & Median & $\mathrm{F}$ & $\mathrm{p}$ & Median & $\mathrm{F}$ & $p$ \\
\hline Basketball & 14 & $\begin{array}{l}1074.43 \pm 11 \\
1.49\end{array}$ & 4.754 & $.014^{*}$ & $\begin{array}{l}1359.36 \pm 20 \\
2.91\end{array}$ & 2.35 & $.108^{*}$ & $\begin{array}{l}2433.79 \pm 27 \\
5.45\end{array}$ & 4.08 & .025 \\
\hline Handball & 14 & $\begin{array}{l}1129.57 \pm 62 \\
86\end{array}$ & & & $\begin{array}{l}1448.93 \pm 15 \\
9.17\end{array}$ & & & $\begin{array}{l}2578.50 \pm 19 \\
2.17\end{array}$ & & \\
\hline Volleyball & 14 & $\begin{array}{l}1209.21 \pm 15 \\
5.51\end{array}$ & & & $\begin{array}{l}1524.93 \pm 23 \\
6.86\end{array}$ & & & $\begin{array}{l}2713.86 \pm 29 \\
8.47\end{array}$ & & \\
\hline Total & 42 & $\begin{array}{l}1137.74 \pm 12 \\
6.48\end{array}$ & & & $\begin{array}{l}1444.4 \pm 208 \\
73\end{array}$ & & & $\begin{array}{l}2575.38 \pm 27 \\
8.23 \\
\end{array}$ & & \\
\hline
\end{tabular}

\section{Results and Discussion}

Considering that volleyball, basketball and handball are team games; players' reaction time being at the top level will provide advantage to the team. While individual skills and technique were of primary concern in the past, today's comprehensive game mentality prioritizes physical values as the first criterion. It was aimed in the study to examine and reveal the differences of sprint reaction and visual reaction times of female athletes at different branches competing in professional leagues.

Visual reaction values in our study were measured as $541.69 \pm 57.00 \mathrm{~ms}$ in basketball players, as $525.60 \pm 31.25 \mathrm{~ms}$ in handball players, and as $575.11 \pm 101.14 \mathrm{~ms}$ in volleyball players. In other words, it was seen that handball players had the best visual reaction time score, while volleyball players had the worst score.

While Aksoy (2012) did not find statistically significant difference between wrestlers and volleyball players in his study, in which he examined the reaction values of wrestlers, volleyball players and football players, he detected statistically significant difference between football players and other branches $(p<0.05)$. Reaction values of football players were detected to be better than volleyball players and handball players. In the measurements performed; reaction time values were measured as $0.164 \pm 0.02 \mathrm{sec}$. in football players, as $0,182 \pm 0,02 \mathrm{sec}$. in volleyball players, and as $0,185 \pm 0,01$ sec. in wrestlers. In other words, it was seen that football players had the best reaction time score, while wrestlers had the worst score. Despite the branch difference in this study, football and handball spots show similarity in terms of speed and instant changes of direction. Therefore, it supports our study.

When we examine the results of the sprint reaction test we conducted, sprint reaction values of the athletes distributed normally. Athletes' spring reaction total mean values were different from each other $(\mathrm{p}<0.05)$.

In sprint reaction total mean values, basketball players' sprint reaction values were detected to be the best, while volleyball players' to be the worst. There is no relation between the visual reaction and spring reaction measurements of basketball, handball and volleyball players.

In his reaction time test study he performed on the handball goalkeepers of the A National Team, Aşçı (2013) detected that the reaction time of the goalkeepers was lower than the players'; however, these values were statistically insignificant $(\mathrm{p}<0.05)$. In his study, Aşç detected the visual reaction time of handball goalkeepers are $189.9 \mathrm{~ms}$, and of field players as $204.8 \mathrm{~ms}$. The reason why these values were higher than our study is that handball goalkeepers require reaction times against sudden and fast shots more than other positions.

Polat (2000) compared the reaction times of table tennis players and sedentaries and found a statistically significant difference in right hand reaction times of table tennis players against light. In their different studies, Singer (1980), Magil (1980) and Schmidt (1988) have detected that the fastest reaction time occurs in cases requiring only a stimulant and reaction. However, the desire to give multiple reactions to multiple stimulants increases when selection of reaction time is of question. Hence, reaction time extends. In their study in which they compared the simple reaction times of two groups that play and do not play basketball, Ün and Erbahçeci (2001) detected that the group playing basketball had shorter reaction times compared to the group that does not play basketball.

In their research in which they examined the agility literature, Sheppard et al (2006), gathered the general agility components under the titles of running with change of direction and perceptual and decision-making factors. It was stated that there is a factor affecting the agility of visual scanning under the factors of perception and decision-making. Medium level, positive and statistically significant relation was detected in this study between the visual reaction time and agility capacity ( $\mathrm{r}=0.455 ; \mathrm{p}<0.05)$. Reimersa and Elizabeth (2011) showed an interaction between the number of tries for reaction time and sex, and detected that women were faster than men against an obstacle despite the fact that they were slower than men in the beginning. In his study, Norrie (1967) stated that the reaction times of the subjects had dropped from $252 \mathrm{~ms}$ to $200 \mathrm{~ms}$ in the first ten trials. Literature shows similarity with our study. Performance level increases as the number of 
repetitions increases in reaction time measurements of the subjects also in our study. The reason for this can be expressed as the fact that attention concentrates more and learning becomes more effective with repetition.

There are very few studies in the literature examining the relation between the measurement times of sprint reaction and visual reaction. Hasdemir et al (2003), examined the reaction times of female handball players according to their positions and recorded visual reaction times as $310 \mathrm{~ms}$ for middle playmakers, $320 \mathrm{~ms}$ for centres, and as $310 \mathrm{~ms}$ for wingers. Although this study is better than ours, the reason for it may be the fact that we did not make any grouping by positions.

In the study he performed on the athletes in Spanish National Teams of Fencing and Karate, Di Russo et al (2006), detected the reaction time mean of male fencers as $356.49 \pm 38.50 \mathrm{~ms}$, reaction time mean of female fencers as $397.11 \pm$ $32.11 \mathrm{~ms}$, reaction time mean of male karate players as $399.00 \pm 72.50 \mathrm{~ms}$, and reaction time mean of female karate players as $396.84 \pm 30.23 \mathrm{~ms}$. While no significant difference was found between the reaction times of female fencers and female karate players, meaningful difference was found between male fencers and male karate players as a result of the study $(\mathrm{p}<0.05)$. Reaction time values recorded in this study are in line with our study. This difference is considered to arise from branch-specific motoric properties.

According to the studies of Kashihara and Nakahara (2005), reaction time increases after an intense exercise; however, this speed can be detected within the 8 minutes following end of exercise. In this test, reaction time returns to its old values after $8-10$ minutes.

It is evident that reaction time is an important property playing role in branches of sport which require sprint. Speed, which is a required motoric property for athletes, requires not only agility but also instant stopping, re-acceleration and changing direction in basketball, volleyball and handball. In addition, requirement of short-distance runs within competition, the struggle to intercept the ball before the opponent, and fast offense organizations may lead us to think that reaction time is important in basketball, volleyball and handball.

The research conducted by John et al., in which they searched for the reliability and similarity between different measurements of speed and agility using a commercially available timing gate system, is the first study according to the authors that has been performed to display the reliability of sensitive agility upon multiple trials. At the end of this study, they detected that exercise stages were not required while using the protocol defined to measure the sensitive agility in random change upon absence of change when systematic tendency and previous trials were considered (Oliver and Meyers, 2009). In an agility test containing findings specific to a sport, Gabbet expressed that $3 \%$ mean for sensitive agility test conforms to the mean $\mathrm{CV}$ value, which is $2.8 \%$, following two trials. Although random change was observed when only the following two trials were taken into consideration; he found the reliability of flat and planned agility sprints to be similar with the previous study at the same time (Gabbett et al, 2008).

\section{Conclusion}

It was aimed in this study to compare the sprint reaction and visual reaction times of professional female athletes in different branches, and it can be said as a result that physical properties of athletes came to the forefront in inter-branch sprint reaction measurement. On the other hand, no prominent property of athletes by branches was encountered in visual reaction measurements.

\section{References}

Aagard, P., Simonsen, E. B., Andersen, J. L., Magnusson, P., \& Dyhre-Poulsen, P. (2002). Increased rate of force development and neural drive of human skeletal muscle following resistance training. Journal of applied physiology, 93(4), 1318-1326. https://doi.org/10.1152/japplphysiol.00283.2002

Aksoy, Y. (2012). Comparison of Sprint Reaction Time and Anaerobic Strength of Young Football, Volleyball and Wrestlers. Master Thesis, 36.

Aşç1, A. (2013). Agility training in children Hacettepe University School of Sport Sciences and Technology (internet access).

Bayar, P., \& Koruç, Z. (1992). Preliminary study for determination of Turkish norms of two vehicles measuring reaction time and hand-eye coordination. II. Sports Sciences National Symposium, Ankara, 130-143.

Di, R. F., Taddei, F., Apnile, T., \& Spinelli, D. (2006). Neural correlates of fast stimulus discrimination and response selection in top-level fencers, Neurosci Lett., 408(2), 113-118. https://doi.org/10.1016/j.neulet.2006.08.085

Dündar U Training Theory. (2007). Bullish publishing house, 5th edition, Ankara.

Gabbett, T., Kelly, J. N., \& Sheppard, J. M. (2008). Speed, change of directions peed, and reactive agility of rugby league players. $J$ Strength Cond Res., 22, 174-181. https://doi.org/10.1519/JSC.0b013e31815ef700 
Guyton, A. C., Hall, J. E., Zocchi, L., \& Aicardi, G. (2006). Fisiología médica, 11.

Hasdemir, S., Gündüz, N., \& Müniroğlu, S. B. (2003). The Examination Of The Differences The Visual And Ouditary Reaction Times Of Female Handball Players At Different Positions, Spormeter, 1(1), 49-52.

Kashihara, K., \& Nakahara, Y. (2005). Short-term effect of physical exercise at lactate threshold on choice reaction time. Perceptual and Motor Skills, 100(2), 275-291. https://doi.org/10.2466/pms.100.2.275-291

Magil, R. A. (1980). Motor learning. USA. Wm. C.Brown Comp. Pub., 14, 1-10.

Negrete, R., \& Brophy, J. (2000). Predicting performance of single leg hop, single leg vertical jump and speed and agility test using isokinetic devices. J Sport Rehab, 9, 513-518.

Norrie, M. L. (1967). Practise Effects on Reaction Latency for Simple and Complex Movements. Research Quarterly, $38,79-85$.

Oliver, J. L., \& Meyers, R. W. (2009). Reliability and generality of measures of acceleration, planned agility, and reactive agility. International journal of sports physiology and performance, 4(3), 345-354. https://doi.org/10.1123/ijspp.4.3.345

Polat, Y. (2000). Impact of Quick Force and Sprint Training on Reaction Time. MSc. Konya: Selcuk University.

Reimersa, S., \& Elizabeth, A. (2011). Gender effects on Reaction Time Variability and trial-to-Trial performance, Maylora University of Warwick, United Kingdom.

Renklikurt, T. (1974). Training Information Physical Condition Manual.

Schmidt, R. A. (1988). Motor control and learning: a behavioral emphasis. USA: Human Kinetics pub. Inc.

Sevim, Y. (2006). Handball Technical-Tactical. Ankara: Nobel Publishing House.

Sheppard, J. M., \& Young, W. B. (2006). Agility literature review: Classifications, training and testing. Journal of Sports Sciences, 24(9), 919-932. https://doi.org/10.1080/02640410500457109

Sheppard, J. M., Young, W. B., Doyle, T. L. A., Sheppard, T. A., \& Newton, R. U. (2006). An evaluation of a new test of reactive agility and its relationship to sprint speed and change of direction speed. Journal of Science and Medicine in Sport, 9, 342-349. https://doi.org/10.1016/j.jsams.2006.05.019

Singer, N. R. (1980). Motor learning and human performance. New York. Macmillan Publishing, 26-32.

Tsitskaris, G., Theoharopoulos, A., \& Garefis, A. (2003). Speed, speed dribble and agility of male basketball players playing in different positions. Journal of Human Movement Studies, 45(1), 21-30.

Ün, N., \& Erbahçeci, F. (2001). The Evaluation of Reaction Time on Mentally Retorded Children. Pediatric Rehabilition, 4(1), 17-20. https://doi.org/10.1080/13638490151068410

Üstdal, K. M., \& Köker, A. H. (1998). How to Earn Sporda High Performance. Istanbul, Nobel Medical Bookstores.

Young, W. B., McDowell, M. H., \& Scarlett, B. J. (2001). Specificity of sprint and agility training methods. J.Strength Cond Res., 15, 315-319. https://doi.org/10.1519/00124278-200108000-00009

\section{Copyrights}

Copyright for this article is retained by the author(s), with first publication rights granted to the journal.

This is an open-access article distributed under the terms and conditions of the Creative Commons Attribution license which permits unrestricted use, distribution, and reproduction in any medium, provided the original work is properly cited. 